\title{
BMJ Open Impact of the great recession on self- perceived health in Spain: a longitudinal study with individual data
}

\author{
Marc Saez, ${ }^{1,2,3}$ Joaquim Vidiella-Martin, ${ }^{4,5}$ Guillem López Casasnovas ${ }^{3,6,7}$
}

\begin{abstract}
To cite: Saez M, VidiellaMartin J, Casasnovas GL. Impact of the great recession on self-perceived health in Spain: a longitudinal study with individual data. BMJ Open 2019;9:e023258. doi:10.1136/ bmjopen-2018-023258

- Prepublication history for this paper is available online. To view these files, please visit the journal online (http://dx.doi. org/10.1136/bmjopen-2018023258).
\end{abstract}

Received 28 March 2018 Revised 10 August 2018 Accepted 27 September 2018

Check for updates

(C) Author(s) (or their employer(s)) 2019. Re-use permitted under CC BY-NC. No commercial re-use. See rights and permissions. Published by BMJ.

For numbered affiliations see end of article.

Correspondence to

Dr Marc Saez;

marc.saez@udg.edu

\section{ABSTRACT}

Objectives Our objective in this study is to evaluate the impact the Great Recession (2008-2014) had on selfperceived health in Spain.

Design We use a longitudinal database (four waves of the Bank of Spain's Survey of Household Finances (2005, 2008, 2011 and 2014)) with repeated observations of the same individuals before and after the Great Recession. Interventions We consider the Great Recession in a natural experiment and we introduce it as an explanatory variable in a mixed logistic regression model in which we explain the probability of a subject declaring poor health (fair, bad and very bad). In the model we control for both observed and unobserved confounders at both individual and family level.

Results We find an average downward trend in selfperceived health during the most severe period of the Great Recession (2009-2011). However, the fact that the adjusted measures are less volatile than the crude ones shows that variation in health status can be captured by either demographic or socioeconomic controls. In fact, there are significant differences in the impact the economic crisis had on health in terms of gender and age group. In particular, the (adjusted) risk of declaring poor health increases after the crisis began but only in those families in which the reference person is a woman younger than 45 years of age or a man aged 75 years or older.

Conclusions Given our results, we discuss the link between financial wealth and self-rated health and how policy-makers could address the health inequalities that arise from adverse economic and financial shocks.

\section{INTRODUCTION}

It is widely acknowledged that the Great Recession (2007-2013) and the period of general economic decline that followed it had an adverse impact on health. In January 2018 we carried out a PubMed database search for studies published in English between January 2008 and December 2017 that assessed these effects. By combining the keywords health, economic crisis, economic downturn and financial crisis, we found 1760 studies, including 211 systematic literature reviews.

The consequences the economic crisis had for health essentially encompassed an increase in mental health issues and suicide

\section{Strengths and limitations of this study}

First, we document the asymmetries in the health impact the Great Recession had, but we do not deal with their subsequent consequences.

- Second, we analyse self-perceived health rather than objective health (morbidity or mortality).

- We make use of a longitudinal database with repeated observations of the same individuals before and after the crisis.

- We control not only for observed confounders at the individual and at the family level but also for the unobserved ones.

- Finally, we use weights to recover the randomness of the sample, which is representative of the Spanish population as a whole.

rates, as well as some other effects related to the rapid rise in unemployment and the worsening economic conditions. Unemployment rates are frequently used as a proxy for the business cycle when assessing the effect of macroeconomic fluctuations on mortality rates. When analysed at country-level, mortality has been found to decrease in times of economic recession. However, individual-level studies have found risk of death to be larger among unemployed relative to their employed counterparts. This paradox is explained by simultaneous causality between ill-health and unemployment. ${ }^{1}$

In some specific countries (the so-called PIGS: Portugal, Italy, Greece and Spain), the consequences for healthcare have been more prevalent. These countries experienced major cutbacks in public health expenditure, reductions in the numbers of their healthcare professionals, as well as salaries and pensions, a decline in the public procurement of medical goods, rapid reforms in the pharmaceutical and social insurance sectors and increasingly inadequate primary care services. In the meantime, there has been a greater demand for public healthcare services coupled with increasing expectations of it. Consequently, this has led 
to deteriorating access to healthcare, increasing out-ofpocket contributions and growing monitoring and efficiency concerns. ${ }^{2}$

It is evident that the issues being confronted during the economic crisis magnified the problems and negative social norms and attitudes that already existed. For instance, patient dissatisfaction with long waiting lists, burnout among health professionals and the political utilisation of the healthcare system for electoral purposes. Public confidence in formal institutional networks such as governments, political parties and public institutions has been declining since the debt crisis commenced, partly because the public blames Europe and the political parties in power for the economic situation they find themselves in. Apart from the findings that emerged from the systematic reviews, it also became clear that greater attention should be paid to the way health information is transmitted to the public (ie, 'health literacy'), as this affects health outcomes and behaviour, along with overall health expectations. ${ }^{3}$

While self-rated health may be one of the most widely studied health indicators in the literature, the results are usually inconsistent. Even though most studies find that the Great Recession increased the risk of declaring poor health (ie, fair, bad or very bad health), ${ }^{4-13}$ there are yet others that find the opposite. ${ }^{14-21}$ In a recent systematic review, Parmar et al, point out that such heterogeneity in the results could be attributed to the country and group analysed.$^{22}$ However, much, if not all, of the heterogeneity could also come from methodological errors. In fact, only 5\% (two out of 41) of the studies that Parmar et al, reviewed were rated as being 'strong' and with a low risk of bias in the overall risk assessment they performed. As such, Parmar et al, recommend caution when interpreting the results. ${ }^{22}$ Among the 12 studies they reviewed ${ }^{4-10} 14-18$ that assessed the effects the crisis had on self-perceived health, only one was rated as having a low risk of bias. ${ }^{9}$ Furthermore, four of the five studies concluding that the crisis decreased the probability of declaring poor health were rated as having a high risk of bias. ${ }^{14-1618}$ The remaining study was rated as a moderate risk. ${ }^{17}$

The most significant biases are those associated with the problems of evaluation ${ }^{23}$ (paradoxically not indicated directly in Parmar $e t a t^{22}$ ). This is a consequence of using observational data from non-experimental designs. Although with some exceptions, these studies use cross-sectional data (health surveys) from before and after the crisis to assess its effects. However, as the individuals interviewed before and after the crisis are not the same, the groups are not comparable. In fact, even if the overall risk of declaring poor health declined over time, when these studies did evaluate specific groups (ie, individuals with low levels of education, ${ }^{1420}$ people affected by foreclosure or eviction or at risk of foreclosure or eviction, ${ }^{1924}$ the unemployed,${ }^{21}$ immigrants ${ }^{18}$ or older individuals ${ }^{20}$ ), the risk actually increased. This is because the members of these groups, despite not being the same individuals as before and after the crisis, share some common characteristics and therefore are more comparable. In fact, this raises the problem of a lack of control for confounding (as is explicitly indicated in Parmar et $a l^{22}$ ).

To control for this bias, several strategies can be followed. One way to make the groups comparable is to match individuals from both groups (before and after the crisis). For the Spanish case and using the same data as the majority of studies that find a decrease in the risk of declaring poor health $\operatorname{did}^{14-20}$ (the 2006 and 2011-2012 Spanish Health Survey in both cases, and the 2006 and 2011-2012 Catalan Health Survey in the second case), Urbanos-Garrido and López-Valcárcel ${ }^{25}$ and Arroyo et $a l^{26}$ matched the individuals. They found that although the crisis did not alter the likelihood of reporting poor health for the general population, ${ }^{26}$ unemployment had a significant negative impact on self-rated health, particularly for the long-term unemployed. ${ }^{25} \mathrm{~A}$ second strategy is to explicitly control for the maximum number of confounders possible. Pérez-Romero $\mathrm{et} \mathrm{al} \mathrm{did} \mathrm{just} \mathrm{that.}$ Using the Spanish Health Survey for 2006 and 2011-2012 and including social support variables (among others), they found that the crisis did not change the probability of declaring poor health. ${ }^{27}$ Finally, a third strategy is to use a different design, specifically a longitudinal one with repeated measurements of the same individuals before and after the crisis, so that the individual is their own control (before the crisis). This is the strategy followed by the studies using longitudinal data from the European Union Statistics on Income and Living Conditions (EUSILC) survey ${ }^{4-6} 81213$ as well as other longitudinal data sources (the Greek Longitudinal Labour Market Study $(\text { LLMS })^{9}$ and the World Values Survey (WVS) $)^{11} 28$ that find that the crisis increased the risk of declaring poor health.

Our objective in this study is to evaluate the impact the Great Recession had on self-perceived health in Spain. We use a longitudinal database with repeated observations of the same individuals before and after the crisis and we also control for both observed and unobserved confounders at individual and family levels.

\section{METHODS \\ Design}

We use four waves of a longitudinal database: the Survey of Household Finances (EFF in Spanish) from the Bank of Spain. ${ }^{29}$ Two waves are from before the Great Recession (2005 — carried out between 2002 and 2004-and 2008carried out between 2005 and 2007-), one during the recession (2011—carried out between 2008 and 2010) and the other at the end of it (2014 - carried out between 2011 and 2013). Each wave consists of a random sample of the Spanish population stratified by sex and age.

Spain went into recession twice. The first recession began in the third quarter of 2008 and ended in the first quarter of 2010 and then the double dip began in the second quarter of 2011 and continued up to the third quarter of 2013.

The EEF is a large database designed to synchronise with all the countries in the Euro area system. It provides 
detailed information on the income, assets, debts and spending of Spanish household units. It also contains sociodemographic information and some health indicators, among which is self-rated health. There are two main reasons why we chose the EFF. The first is its longitudinal nature. The EFF samples contain a common subset of households observed at various points in time. The second reason is that it is the only source of data that provides information on the wealth of Spanish families over time. Wealth is an important variable here. In particular, net wealth (ie, assets minus debts) not just in absolute terms, but according to its composition (housing or financial assets) when subjected to different random shocks and risk premia. Household wealth is, in this sense, a kind of buffer that can make family income more resilient to shocks and may also delay the direct financial consequences the crisis might have on personal anxiety and family bankruptcy. Wealth is among the observed confounders we control for and we find it to be a significant determinant of self-assessed health status when we stratify the model for demographic groups and when we do not. We also find wealth to have significant explanatory power over self-rated health when we include it aggregating over sources of wealth (ie, including only net wealth) or when we detail different sources of wealth (real estate, financial and others).

Our sample only includes the members of those families who were interviewed in at least two waves of the EFF and were interviewed both before and after the crisis. In the sample, we included a total of 28678 individuals belonging to 10586 families. This random sample represented a population of 20038899 individuals and 7109404 families.

\section{Variables}

\section{Response variable}

To construct our response variable 'poor health', we used the question, 'How would you describe your health status in general?' Answers could range from 'very good', 'good', 'fair', 'bad' and 'very bad'. Next, we grouped the responses into two categories: fair, bad and very bad (this took the value 1 ) and very good and good (value 0 ).

\section{Explanatory variables}

Our explanatory variable of interest is the year of the survey wave (2005, 2008, 2011 and 2014). Using a smoothing spline, we allow the relationship between the explanatory variable of interest and the response variable to be non-linear. In particular, we include a random effect, associated with the year of the wave, using a random walk of order 1 as a smoother.

As the explanatory variables of control, we include variables at the family level:

1. Gross wealth, without stratifying (categorised in deciles, once trimmed-the first decile was the reference category), and stratified in: (a) real-estate wealth (categorised in deciles, once trimmed-the first decile was the reference category), (b) financial wealth (categorised in deciles, untrimmed-the first decile was the reference category) and (c) real wealth other than real estate (categorised into quintiles, untrimmed - the first quintile was the reference category).

2. Total debt when identifying 'net wealth' (categorised in total debt equal to 0-reference category-and then in quartiles of total positive debt greater than 0 ).

3. Income (categorised in deciles, untrimmed- the first decile was the reference category.

4. Savings rate (categorised in quintiles, once trimmedthe first quintile was the reference category).

5 . Number of family members.

6. Number of family members who work and

7. Property regime of the family dwelling (not owned by the family-reference category- or owned by the family).

We also include an estimate for the savings rate of the household. This variable is the only one not taken directly from the survey. Instead, we approximate it by using information about household income and spending on durables and consumption goods. We inflate this household spending by a factor such that the aggregate savings rate (ie, net income over income) matches the economy saving rates (as calculated by the European Central Bank). Our results are robust to the exclusion of the savings rate; thus the concern about measurement error is mitigated.

We also include control variables at the individual level: sex (taking man as a reference category), age (categorised as younger than 35 years-reference category- 35 to 44 years, 45 to 54 years, 55 to 64 years, 65 to 74 years, 75 years or older), educational level (categorised as insufficient instruction, ie, illiterate, without studies or incomplete primary-reference category-primary, secondary, including vocational training, and university), occupation (working as an employee-reference category—self-employed, unemployed, retired, disabled, student, home maker or other situations), marital status (single (reference category), married or with a partner, divorced or separated and widowed).

In some of the variables of control at family level, there were some very extreme outliers (both left and right), which is why we trimmed them before categorising them, thus excluding $2.5 \%$ of the values of the distribution of the variable both left and right.

\section{Patient and/or public involvement}

It should be noted that neither patients nor the public were involved in this research.

\section{Statistical analysis}

We specify a generalised linear mixed model with binomial response and a logistic link:

$$
\log \left(\frac{\operatorname{Prob}\left(Y_{i j}=1\right)}{1-\operatorname{Prob}\left(Y_{i j}=1\right)}\right)=\eta_{i j}
$$

where $Y$ denotes the response variable (1 for poor health, 0 otherwise), the subscript $i$ denotes the study 
subject, $j$ the family to which the subject belongs and $\eta_{i j}$ a linear predictor for subject $i$.

In the linear predictor for each subject in the model $\{1\}$, we incorporate the variables that might explain the probability of declaring poor health, the explanatory variables described above (ie, observed confounders) as well as two unstructured random effects to control for unobserved confounders. In particular, we considered individual heterogeneity, associated with each individual, and familiar heterogeneity, associated with the family to which the individual belonged.

However, the distribution of wealth is heavily skewed. For this reason, the EFF oversamples the wealthiest households. This is done to ensure that its sample is representative not only of the Spanish population as a whole, but also of the aggregate wealth of the Spanish economy. We corrected that oversampling by including in the models the weights provided by the EFF itself in each of its waves.

We estimate a model $\{1\}$, both without stratifying and stratifying by the sex and the age group (younger than 35 years, 35 to 44 years, 45 to 54 years, 55 to 64 years, 65 to 74 years, 75 years or older) of the reference person of the family.

Given the complexity of our model, we perform inferences using a Bayesian framework. In particular, we followed the integrated nested Laplace approximation (INLA) approach, ${ }^{30}$ within a (pure) Bayesian framework. All analyses were made with the free software R (V.3.4.1), ${ }^{31}$ through the INLA package. ${ }^{30} 32$

\section{RESULTS}

We summarise the results by analysing the crude OR, without adjusting in a model (crude OR from here on) and adjusting in a model (adjusted OR henceforth), for both the whole and the stratified samples. Here, both ORs compare the risk of declaring poor self-rated health relative to the average risk over the whole period. Hence, an increase (decrease) in the OR implies larger (smaller) risk of declaring poor self-rated health compared with the average probability of declaring poor self-rated health over the four waves of the EFF survey.

We first analyse how the reporting of poor self-rated health evolves for the whole sample of individuals (figure 1). The risk of reporting poor self-rated health decreased during the years previous to the crisis (2005 to 2008) - the real estate bubble period in Spain-and later peaked in 2011 (the worst moment of the crisis with a very demoralising double dip recession), before it dropped below pre-crisis values in 2014. When we control for the confounders in our model, the trend persists but is much closer to 1 (ie, the average of the whole period of study). Hence, the first conclusion from our model is that changes in health status in Spain are partly explained by demographic and socioeconomic factors.

A stratified analysis based on the age and gender of the reference person of the family reveals significant differences in the reporting of poor self-rated health trends. We consider six age categories in our analysis. The evolution of the crude OR by age group of the reference person is summarised in figure 2 . We find striking differences both across (ie, by age) and within groups (by gender).

While the crude OR remains virtually unchanged in households where the reference is a person younger than 35 years old, the other five groups experience significant variation over time. When we adjust, our results change upwards to some extent (figure 3). It is worth noting that

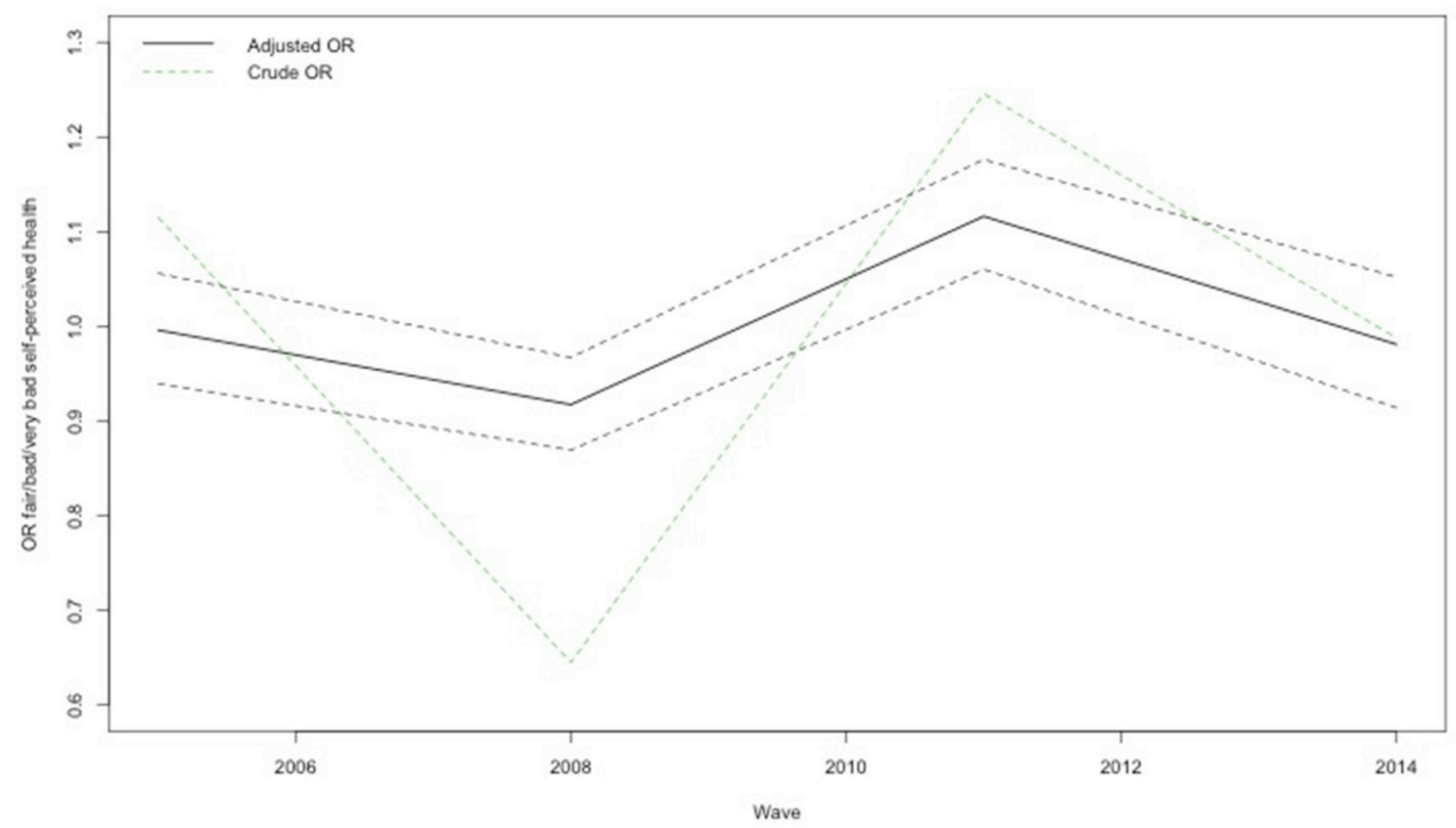

Figure 1 Evolution of self-declaration of poor health (fair/bad/very bad), Spain, 2005-2014. Together with the adjusted OR, its $95 \%$ credibility interval has been drawn. 

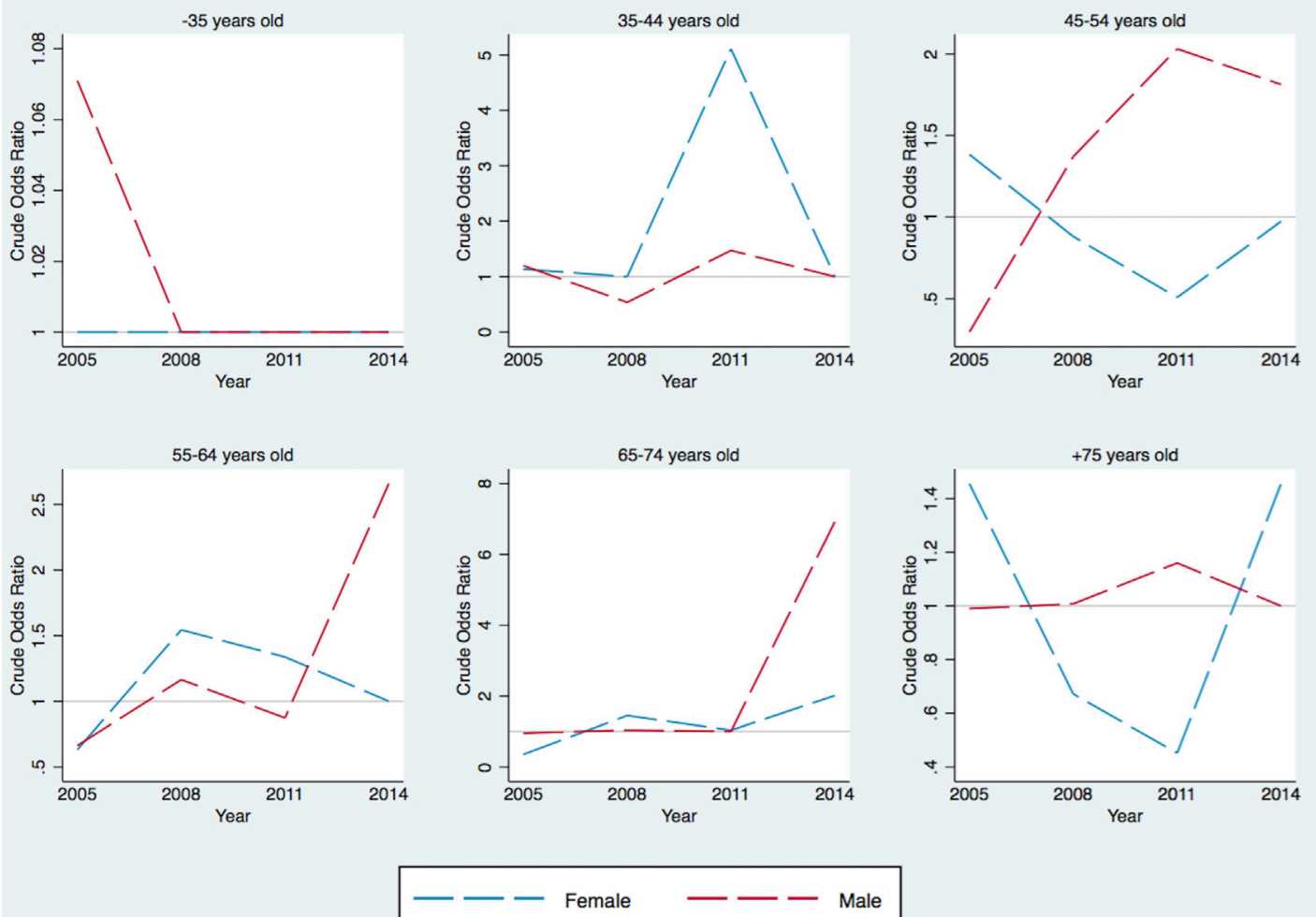

Figure 2 Evolution of self-declaration of poor health (fair/bad/very bad), Spain, 2005-2014, stratified by the sex and age group of the reference person of the household.
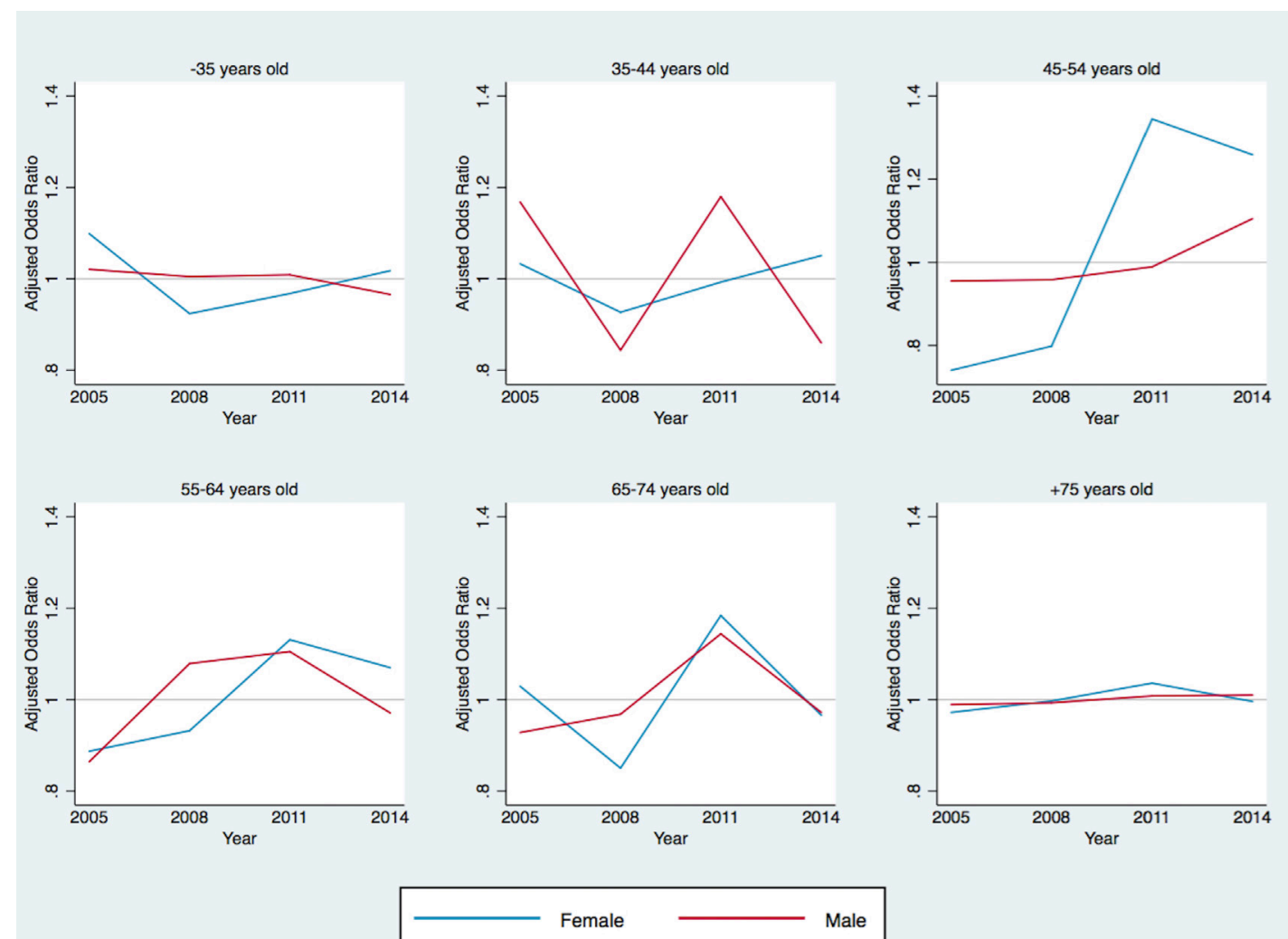

Figure 3 Evolution of the adjusted self-declaration of poor health (fair/bad/very bad), Spain, 2005-2014, stratified by the sex and age group of the reference person of the household. 
for all groups, except the youngest, the adjusted OR is notably closer to 1 , implying that a remarkable share of the variation in the outcome variable was captured by the variables in the model.

In households where the reference person is between 35 and 44 years old, self-assessed health declines at the beginning of the economic crisis and then remains stable for the remaining two waves. When adjusting for the factors in our model, we find that female health recovers steadily, while in those households where the reference person is a man, self-rated health experiences a minor downturn. In both cases, the crude and adjusted ratios are close to average (1), thus implying little volatility in health for our reference group.

Households in which the reference person falls into our third age category (45-54 years old) reflect opposite trends. Individuals living in families where the reference person was a woman had a higher relative risk of declaring poor self-rated health before the crisis (2005). Their crude OR decreased steadily until 2011, before reaching average (1). Meanwhile, for households where the reference person is a man between 45 and 54, the risk of declaring poor self-rated health was lower in relative terms in 2005, and became almost four times larger in 2011. When we adjust (adjusted OR), men experience almost no change during the period, whereas women show higher risk after 2008.

Adults who are close to retirement (ages 55-64) display some of the most relevant findings. Counteracting what we find for the rest of the groups, the crude OR increases from 2005 to 2008 for both genders. While females recover from this, male self-rated health worsens in 2014. When adjusting the OR, we find male self-rated health returns (slightly better) to the period average, whereas women report worse self-rated health.

Those individuals who entered retirement age right before the crisis (ages 65-74) show a surprising peak in the crude OR in the economic recovery period (2014). The trend is common in both females and males, although it is significantly more pronounced for the latter group. Despite this fact, the trend is reversed when we adjust. For both genders, the adjusted OR is similar to the average population pattern and shows the recurring minimum value in 2008 and maximum value in 2011, implying that self-rated health was better right at the end of the boom/ start of the crisis and worsened in the toughest years for the Spanish economy. Also, the fact that the crude OR peaks in 2014 with such extreme values suggests that the financial shock for this age group (captured by our model) was significantly larger than for other groups.

In the oldest age group (older than 75 years), men and women once again exhibit opposing trends. While female individuals had a lower probability of declaring poor selfrated health during the economic and financial crisis, males showed a higher risk. However, virtually all of this variation is captured by the variables in our model and the adjusted OR remains approximately flat; around 1 for the four waves.

\section{DISCUSSION}

In this paper we document the uneven health impact the economic and financial crisis that affected Spain from 2008 to 2014 had on individuals and groups. We make use of the Bank of Spain's Household Financial Survey, which is a representative sample of the whole population followed over four waves (2005, 2008, 2011 and 2014). We obtain detailed information on individuals' socioeconomic conditions as well as on their self-rated health. We summarise our analysis using two results that show the risk of declaring poor self-rated health for each group studied in relation to the average of the 2005-2014 period. Our first measure is the crude OR, that is, the unadjusted risk of reporting poor self-rated health. The second and main methodological contribution of this paper is the adjusted OR, which corrects the dependent variable of the model (risk of reporting poor self-rated health) taking into account socioeconomic individual controls, household financial portfolios, family income levels as well as individual and family heterogeneities.

Summing up, the main takeaways of the paper are threefold. First, we document an average downward trend in self-perceived health during the most severe period of the Great Recession (2009-2011). The recovery of the economy coincides with an improvement in self-perceived health, suggesting a close relationship between the two; this also occurs at the macroeconomic level. Second, the fact that the adjusted measures are less volatile than the crude ones shows that variation in health status can be (at least partially) captured by either demographic or socioeconomic controls. Third, we document significant differences in the impact the economic crisis had on health, showing that not all age/gender groups suffered the same consequences. In particular, and unlike the average evolution for the whole sample, the (adjusted) risk of declaring poor health increases after the crisis begins only in those families in which the reference person is a woman younger than 45 years of age or a man aged 75 years or older.

While the aim of this paper is to document health inequalities arising from the financial crisis and not to find causal links, in this section we discuss where our evidence points to.

Several finance-related mechanisms may be behind our findings. Net wealth fluctuates little during the crisis (well below income). Between 2008 and 2014, households where the reference person is a man aged between 45 and 54 are those most severely punished by the crisis in terms of net wealth (probably due to the overburden of the housing debt), but not as much on income levels. Young cohorts do not lose very much during the crisis since they have little accumulated wealth prior to the start of it. Meanwhile, pensioners even increase their net wealth (up to $50 \%$ ). That said, there is a significant reduction in income ( $15 \%$ to $20 \%$ ) for all non-pensioners, and particularly for those between 45 and 65 years old. This leaves the 45-year-old to 55-year-old group in the more precarious financial situation, something that is reflected 
in their increased risk of declaring poor health status even after the economic recovery. Therefore, we find suggestive evidence that accumulated financial wealth is used as a buffer to offset adverse financial shocks, and self-perceived health deteriorates when such a buffer is sharply reduced.

In fact, this reduction could explain the deterioration of self-perceived health, apparent even after the crisis, in the two population groups that behave differently from the rest (ie, families in which the reference person is a woman under 45 years of age or a man aged 75 years or more). It is worth noting that the EFF determines that a woman is the reference person of the family only when there is no adult man in the family. Thus, these families are mainly comprised either of single women (younger than 35 years of age) or separated or divorced women (between 35 and 44 years old) and, in both cases, with children who have not yet entered the labour market. These are the families in the most precarious financial situation as their median gross wealth is $€ 192212$ (€223144 in the case of a family where the reference person is a man younger than 45 years of age, and $€ 331733$ without stratifying) and the median income is $€ 29331$ ( $€ 35391$ in the case of a family where the reference person is a man younger than 45 years of age, and $€ 36699$ without stratifying). In the second case, the families comprise mainly widowed men who live alone (older than 75 years). In this case, we are dealing with families that, although they have a higher gross wealth than the average (median gross wealth $€ 504854$ vs $€ 331733$ without stratifying) they have the second lowest income (median gross wealth $€ 31338$ vs $€ 36699$ without stratifying), ie, behind the families in which the reference person is younger than than 45 years.

Some of the clues in analysing how and why gender/age groups have been affected differently by the crisis include (1) the personal cost of maternity for women (given the precariousness of most female jobs, becoming a mother usually results in job loss) and (2) the lack of job opportunities for women (despite the evidence that a second wage is an important shelter against family poverty), as well as the fact that (3) the overall rate of unemployment doubled for young individuals and (4) the middle-aged long-term unemployed had few prospects of re-entering the labour force during the crisis. On the other hand, pensioners bucked this trend by maintaining their pension (on average more than those actuarially fair) and enjoying an increase in their pensions during the period of low inflation.

The Spanish social security system is universal and meets its objectives by using public expenditure ratios, both per capita and in terms of GDP figures. It is realistically situated in the lower-middle level among the other European Union member countries. In terms of equity, the gap in socioeconomic inequalities was increased first by immigration, driven by the economic boom at the beginning of the 2000s, and then by the high unemployment rates (mainly during 2008-2014) resulting from the economic crisis. Consequently, vulnerable groups (in particular, the unemployed, children and the elderly population) have been created despite access to universal health services not having changed. Note, however, that there was a short-term reform implemented in September 2012 that restricted access to public healthcare for undocumented immigrants.

However, universalism is not itself a resilient enough shelter for the economic crisis as it distributes its consequences unevenly. Actual utilisation is ultimately the leading vector to signal the redistributive impact a universal healthcare system has. In view of that, it is important to determine how Spanish healthcare system financing and use changed during the recent economic crisis. So far, aggregate data tell us that little changed. Although the increase in public healthcare spending certainly did slow down, there were very few items that were directly affected. So why then, was the population's health (on average) reduced? This can be explained by the fact that the greatest burden of the reduction in healthcare spending has been borne by Spanish healthcare professionals as they shouldered wage and staff freezes and restrictions on drug expenditure. This is short-term fix, based on pressuring healthcare workers to be more productive and lowering unit costs. Nonetheless, the impact the reduction in healthcare finance has had on the utilisation of services for some social groups is less known and the Spanish perception of their self-assessed health has suffered as a result.

Our study has some limitations. First, while we document the asymmetries in the health impact the Great Recession had, we do not deal with their consequences. Wealth inequality is much more pronounced than income inequality in Spain. With the crisis, wealth (a stock variable) should fluctuate relatively less than income (flow variable). We observe the former factor in the difference between the mean and median values for most of the sample, particularly among 45 years and older, and the latter in the increasing SD for the net wealth, mostly for the oldest groups. Such differences are, however, less likely to be as sensitive to health as in the younger groups due to economic conditions. Wealth inequality may have a more important role on self-rated health, with an increasing impact for those between 35 and 44 years old, and income inequality for women over 75 years and older.

Second, we analyse self-perceived health, not objective health (morbidity or mortality). Although it is true that since this is highly correlated with functional status, and self-perceived health can be considered a proxy for objective health, it must be taken into account that it is also related to psychosocial determinants and the socioeconomic position of the individual and possibly even with other factors such as satisfaction with healthcare services. ${ }^{33}$ For this reason, self-perceived health could be much more sensitive than other more objective indicators are.

Third, some authors suggest that ORs may be, in fact, not very informative regarding the interpretation of health inequalities. ${ }^{34}$ 
Our final limitation is methodological because, as in any Bayesian analysis, the choice of the prior distributions of model parameters (ie, priors) may have had a considerable impact on the results. However, we used priors that penalise the complexity (PC priors) ${ }^{35}$ and which have been found to be very robust. Furthermore, we performed sensitivity analyses to assess how the prior on the hyper-parameters influenced the results of the estimation. To do this, we first increased the precision (lowering the variance) and then we tested other priors i.e., those used by default in R INLA ( $\log$ gamma) with different shape and inverse scales: uniform and centred half-normal. In all cases, the PC priors provided better results.

We believe that these limitations are offset by the strengths of our study. For example, we make use of a longitudinal database with repeated observations of the same individuals before and after the crisis so that the subject is their own control and, therefore, the pre-crisis and post-crisis samples are perfectly comparable. Furthermore, we control not only for observed confounders at the individual and at the family level, but also for the unobserved ones. In particular, we consider individual heterogeneity - associated with each individual- and family heterogeneity-associated with the unit to which the individual belonged. Likewise, wealth is among the observed confounders we control for. In fact, household wealth is, in this sense, a kind of buffer that can make family income more resilient to shocks and so may delay the direct financial consequences the crisis has on personal anxiety and family bankruptcy. Finally, we use weights to recover the randomness of the sample, which is representative of the Spanish population as a whole.

Our study provides two key messages for policy-makers. First, the links between wealth and income should not be disregarded when analysing healthcare, neither on their absolute levels, nor on disparities among groups and their evolution over time, since these account for a significant part of the changes in health status. Second, given the observed uneven distribution (by age) of the risk aversion among individuals, policy-makers should design policies targeted to mitigate the impact of the financial crisis (for instance, the loan-to-value housing mortgages that are too high and their resulting effects that lead to poverty). This is reflected by individuals taking on excess debt in terms of lifecycle earnings, particularly those more exposed to income fluctuation. In such cases individuals experience a more serious financial impact from the recession and, consequently, their health is more likely to suffer as well.

\footnotetext{
Author affiliations

${ }^{1}$ Research Group on Statistics, Econometrics and Health (GRECS), University of Girona, Girona, Spain

${ }^{2}$ CIBER of Epidemiology and Public Health (CIBERESP), Madrid, Spain

${ }^{3}$ Center for Research in Health and Economics (CRES), Universitat Pompeu Fabra, Barcelona, Spain

${ }^{4}$ Erasmus School of Economics, Erasmus University Rotterdam, Rotterdam, The Netherlands

${ }^{5}$ Tinbergen Institute, Amsterdam, The Netherlands
}

${ }^{6}$ Department of Economics and Business, Department of Economics and Business, Barcelona, Spain

${ }^{7}$ Barcelona Graduate School (BGSE), Universitat Pompeu Fabra, Barcelona, Spain

Acknowledgements We appreciate the comments from the associate editor and the two anonymous reviewers, which have undoubtedly helped to improve this work.

Contributors GLC and MS had the original idea for the paper. MS designed the study. The bibliographic search and the writing of the introduction were carried out by JV-M and MS. The methods and statistical analysis were chosen and performed by MS. JV-M created the tables and figures. All authors wrote the results and the discussion. The writing and final editing was done by MS, JV-M and GL-C. All authors reviewed and approved the manuscript.

Funding This work was partly funded by the University of Girona (MPCUdG2016 and GDRCompetUdG2017) and by an unrestricted grant from Obra Social 'La Caixa'.

Competing interests The manuscript is an original contribution that has not been published before, whole or in part, in any format, including electronically. All authors will disclose any actual or potential conflict of interest including any financial, personal or other relationships with other people or organisations that could inappropriately influence or be perceived to influence their work, within three years of beginning the submitted work.

Patient consent Not required.

Provenance and peer review Not commissioned; externally peer reviewed.

Data sharing statement We used Survey of Household Finances (EFF in Spanish) from the Bank of Spain. Public and freely accessible at:https://www.bde.es/bde/en/ areas/estadis/Otras_estadistic/Encuesta_Financi/.

Open access This is an open access article distributed in accordance with the Creative Commons Attribution Non Commercial (CC BY-NC 4.0) license, which permits others to distribute, remix, adapt, build upon this work non-commercially, and license their derivative works on different terms, provided the original work is properly cited, appropriate credit is given, any changes made indicated, and the use is non-commercial. See: http://creativecommons.org/licenses/by-nc/4.0/.

\section{REFERENCES}

1. Tapia Granados JA, lonides EL. Population health and the economy: mortality and the great recession in Europe. Health Econ 2017;26:e219-e235.

2. Simou E, Koutsogeorgou E. Effects of the economic crisis on health and healthcare in Greece in the literature from 2009 to 2013: a systematic review. Health Policy 2014;115:111-9.

3. Antunes A, Frasquilho D, Cardoso G, et al. Perceived effects of the economic recession on population mental health, well-being and provision of care by primary care users and professionals: a qualitative study protocol in Portugal. BMJ Open 2017;7:e017032.

4. Zavras D, Tsiantou V, Pavi E, et al. Impact of economic crisis and other demographic and socio-economic factors on self-rated health in Greece. Eur J Public Health 2013;23:206-10.

5. Vandoros S, Hessel P, Leone T, et al. Have health trends worsened in Greece as a result of the financial crisis? A quasi-experimental approach. Eur J Public Health 2013;23:727-31.

6. Ferrarini T, Nelson K, Sjöberg O. Unemployment insurance and deteriorating self-rated health in 23 European countries. J Epidemiol Community Health 2014;68:657-62.

7. Reile R, Helakorpi S, Klumbiene J, et al. The recent economic recession and self-rated health in Estonia, Lithuania and Finland: a comparative cross-sectional study in 2004-2010. J Epidemiol Community Health 2014;68:1072-9.

8. Hessel P, Vandoros S, Avendano M. The differential impact of the financial crisis on health in Ireland and Greece: a quasi-experimental approach. Public Health 2014;128:911-9.

9. Drydakis N. The effect of unemployment on self-reported health and mental health in Greece from 2008 to 2013: a longitudinal study before and during the financial crisis. Soc Sci Med 2015;128:43-51.

10. Huijts T, Reeves A, McKee M, et al. The impacts of job loss and job recovery on self-rated health: testing the mediating role of financial strain and income. Eur J Public Health 2015;25:801-6.

11. Ergin I, Mandiracioglu A. Demographic and socioeconomic inequalities for self-rated health and happiness in elderly: the situation for turkey regarding world values survey between 1990 and 2013. Arch Gerontol Geriatr 2015;61:224-30. 
12. Abebe DS, Tøge AG, Dahl E. Individual-level changes in self-rated health before and during the economic crisis in Europe. Int $J$ Equity Health 2016:15:1.

13. Nelson K, Tøge AG. Health trends in the wake of the financial crisisincreasing inequalities? Scand J Public Health 2017;45:22-9.

14. Rajmil L, Medina-Bustos A, Fernández de Sanmamed MJ, et al. Impact of the economic crisis on children's health in Catalonia: a before-after approach. BMJ Open 2013;3:e003286.

15. Regidor E, Barrio G, Bravo MJ, et al. Has health in Spain been declining since the economic crisis? J Epidemiol Community Health 2014;68:280-2.

16. Aguilar-Palacio I, Carrera-Lasfuentes P, Rabanaque MJ. Youth unemployment and economic recession in Spain: influence on health and lifestyles in young people (16-24 years old). Int J Public Health 2015;60:427-35.

17. Bartoll X, Toffolutti V, Malmusi D, et al. Health and health behaviours before and during the Great Recession, overall and by socioeconomic status, using data from four repeated crosssectional health surveys in Spain (2001-2012). BMC Public Health 2015;15:865

18. Gotsens M, Malmusi D, Villarroel N, et al. Health inequality between immigrants and natives in Spain: the loss of the healthy immigrant effect in times of economic crisis. Eur J Public Health 2015;25:923-9.

19. Rajmil L, Siddiqi A, Taylor-Robinson D, et al. Understanding the impact of the economic crisis on child health: the case of Spain. Int $J$ Equity Health 2015;14:95

20. Barroso C, Abásolo I, Cáceres JJ. Health inequalities by socioeconomic characteristics in Spain: the economic crisis effect. Int J Equity Health 2016;15:62.

21. Calzón-Fernández S, Fernández-Ajuría A, López-del-Amo-González MP, et al. Sex differences of perceived health before and during the economic crisis (2007 and 2011). Spain [in Spanish]. Rev Esp Salud Publica 2017;16:91.

22. Parmar D, Stavropoulou C, loannidis JP. Health outcomes during the 2008 financial crisis in Europe: systematic literature review. BMJ 2016;354:i4588.

23. Mullahy J, Manning W. Statistical issues in cost-effectiveness analysis. Sloan FA, ed. Valuing Health Care. Cambridge: Cambridge University Press, 1996:149-84.
24. Vásquez-Vera H, Rodríguez-Sanz M, Palència L, et al. Foreclosure and Health in Southern Europe: results from the platform for people affected by mortgages. J Urban Health 2016;93:312-30.

25. Urbanos-Garrido RM, Lopez-Valcarcel BG. The influence of the economic crisis on the association between unemployment and health: an empirical analysis for Spain. Eur J Health Econ 2015;16:175-84

26. Arroyo E, Renart G, Saez M. How the economic recession has changed the likelihood of reporting poor self-rated health in Spain. Int J Equity Health 2015;14:149.

27. Pérez-Romero S, Gascón-Cánovas JJ, de la Cruz-Sánchez E, et al. [Economic recession (2006-2012) and changes in the health status of the Spanish population]. Salud Publica Mex 2016;58:41-8

28. WVS, 2018. World Values Survey. http://www.worldvaluessurvey.org/ WVSContents.jsp (accessed 17 Jan 2018).

29. Bank of Spain. Survey of household finances [in Spanish]. https:// www.bde.es/bde/es/areas/estadis/Otras_estadistic/Encuesta Financi/ (accessed 17 Jan 2018).

30. Rue H, Martino S, Chopin N. Approximate Bayesian inference for latent Gaussian models by using integrated nested Laplace approximations. Journal of the Royal Statistical Society: Series B 2009:71:319-92.

31. R Core Team, 2018. R foundation for statistical computing, Vienna, Austria A language and environment for statistical computing. $R$ foundation for statistical computing, Vienna, Austria $2017 \mathrm{https}: / /$ www.R-project.org/ (accessed 16 Jan 2018).

32. Bayesian computing with INLA, 2018. R INLA project, 2017. Available at http://www.r-inla.org/home (accessed 16 Jan 2018).

33. Paul $\mathrm{P}$, Hakobyan $\mathrm{M}$, Valtonen $\mathrm{H}$. The association between self-perceived health status and satisfaction with healthcare services: evidence from armenia. BMC Health Serv Res 2016;16:67.

34. King NB, Harper S, Young ME. Use of relative and absolute effect measures in reporting health inequalities: structured review. BMJ 2012;345:e5774.

35. Simpson D, Rue H, Riebler A, et al. Penalising model component complexity: a principled, practical approach to constructing priors. Statistical Science 2017;32:1-28. 